\title{
Hydrogen FBG Sensor Using Pd/Ag Film With Application in Propulsion System Fuel Tank Model of Aerospace Vehicle
}

\author{
Said SAAD ${ }^{1 *}$, Lotfi HASSINE ${ }^{2}$, and Wassim ELFAHEM ${ }^{3}$ \\ ${ }^{1}$ Group of Electronics and Quantum Physics, Laboratory of Advanced Materials and Quantum Phenomena, Faculty of \\ Sciences of Tunis, 2092, Tunisia \\ ${ }^{2}$ National Institute of Applied Sciences and Technology, University of Carthage, North Urban Center of Tunis-B.P. 676 \\ Cedex Tunis, 1080, Tunisia \\ ${ }^{3}$ Regio iT Gesellschaft mit beschrankter Haftung, Aachen, Germany \\ ${ }^{*}$ Corresponding author: Said SAAD_ E-mail: said.saad@fst.rnu.tn
}

\begin{abstract}
The high efficiency hydrogen fiber Bragg grating (FBG) sensor is presented. The sensitive film was a new alliance of palladium-silver (Pd-Ag). In addition, the titanium (Ti) layer was used as the adhesive layer. The presented sensor showed the resolution of more than $60 \mathrm{pm} / 1 \% \mathrm{H}_{2}$, and a fast response time of $4 \mathrm{~s}-5 \mathrm{~s}$ was guaranteed in the $0.1 \% \mathrm{H}_{2}-4 \% \mathrm{H}_{2}$ range. Moreover, the life time of the sensor was investigated. The obtained results showed that the sensor had an enhanced life time. Furthermore, the sensor was applied in the propulsion system fuel tank model of the aerospace vehicle. The obtained results indicated that it is a prevention system against the disaster aerospace due to hydrogen leakage.
\end{abstract}

Keywords: Hydrogen fiber Bragg grating, palladium-silver, faster response time, propulsion system fuel tank, prevention system, hydrogen leakage

Citation: Said SAAD, Lotfi HASSINE, and Wassim ELFAHEM, "Hydrogen FBG Sensor Using Pd/Ag Film With Application in Propulsion System Fuel Tank Model of Aerospace Vehicle," Photonic Sensors, 2014, 4(3): 254-264.

\section{Introduction}

Actually, hydrogen has become increasingly interesting for the aerospace and energy industries [1], due to its promising properties and high energy content. As an example, liquid hydrogen $\left(\mathrm{LH}_{2}\right)$ is emerging as an alternative fuel for aircrafts. So aerospace vehicle manufacturers are expected to provide sufficient capacity for flight durations, ranging from a few minutes to several days [2]. Nevertheless, using a hydrogen system can be dangerous because hydrogen is easy to leak due to its smallest molecule. Thus, it is required to establish the hydrogen leakage preventive system with sufficient safety. The development of the safety prevention systems is understood, for hydrogen leakage detection like: catalysis, thermal conductivity, electrochemistry, resistance based, working function based, mechanic, optics, and acoustics [3]. All of these systems differ in the measuring range, sensitivity, selectivity, and response time. Especially, the type of the optical system with the FBG technology seems a good result, in particularity for the lower measurement.

We can use the FBG technology as the safety prevention system for hydrogen leakage detection in 
long flight durations and in aging aerospace vehicles. In this paper, our results are based on a special robust FBG sensor with a length of $4 \mathrm{~mm}$ which is developed by our research group. The experimental results of this sensor are presented in [4]. In addition, a new alliance of the Pd-Ag (palladium-silver) material $(500 \mathrm{~nm})$ as the hydrogen sensitive film is used with the titanium (Ti) layer $(35 \mathrm{~nm})$ as the adhesive layer to enhance the strength between the Pd-Ag layer and the optic cladding. From the authors' knowledge, this alliance was never proposed before as the hydrogen sensitive film with the FBG technology. It was presented only with Schottky diode hydrogen sensor by NASA(National Aeronautics and Space Administration) in 1995 [5]. The purpose of this alliance is to obtain the higher sensitivity, faster response time, and further repeatability in the measurement. So the enhancement in life time and location of the leakage positions of hydrogen more quickly and accurately will be obtained. Thus, the faster reparation is possible, and this is very important to save people and equipments. In the first part of this paper, this new sensor is presented. In the second part, the results that describe the sensor sensitivity, response time, and repeatability to hydrogen are investigated. And in the third part, the results in the real-time way of the applications of 3 sensors and the type that is presented in the first part, in the propulsion system fuel tank model of the aerospace vehicle using the selector technique are investigated too. The obtained results confirmed that the proposed sensor is an efficient sensor for the hydrogen leakage detection.

\section{Design of hydrogen FBG sensor}

The FBG technology was created first by Hill et al. in 1978 at the Canadian Communications Research Center (CRC), Otawa, Canada [6]. Afterwards, several researcher groups well developed this technology especially in the last decade. The most distinctive characteristic of the
FBG technology is the flexibility which is offered for achieving desired spectral characteristics. And so, various counting physical parameters can be varied, including: the fiber properties, effective refractive index modulation, FBG length, apodized function, chirp period, and whether the grating ropes reverse propagation or co-propagation at a desired wavelength. And usually, the Bragg grating that is inscribed in the fiber core, acts as a spectral filter that allows a part of the incident signal to be reflected, as shown in Fig. 1. The classic expression that gives the central wavelength of the reflected signal is [7]

$$
\lambda_{B}=2 n_{\mathrm{eff}} \Lambda(z)
$$

where $\lambda_{B}$ is the central wavelength of the spectral band reflected by the Bragg grating. Generally, the Bragg wavelength is nominated. $n_{\text {eff }}$ is the effective refractive index of the optical fiber, and $\Lambda(z)$ is the grating period, obviously being a constant if we have a uniform Bragg grating. In this paper, it is not the case, because we use a special FBG where the grating period is expressed by

$$
\Lambda(z)=\left\{\begin{array}{l}
\Lambda_{0}\left(1-c_{p} z\right) \text { for }\left[0 \ldots \frac{z}{2}\right] \\
\Lambda_{0}\left(1+c_{p} z\right) \text { for }\left[\frac{z}{2} \ldots z\right]
\end{array}\right.
$$

where $\Lambda_{0}$ and $\Lambda_{0}^{\prime}$ are the nominal grating periods, and $c_{p}(\mathrm{~nm} / \mathrm{cm})$ is the linear chirp coefficient. In the previous work of our research group that is presented in [4] as we have talked before, the effectiveness of this special design of the FBG has been shown, in which it gave the robustness, acceptable sensitivities of strain and temperature with the higher stability. With the following parameters: the length of $4 \mathrm{~mm}, \Lambda_{0}=0.53 \mu \mathrm{m}$, $\Lambda_{0}^{\prime}=0.5284 \mu \mathrm{m}, \quad c_{p}=1.5 \mathrm{~nm} / \mathrm{cm}, \quad n_{\mathrm{eff}}=1.456$, and refractive index modulation equal to $2.5 \times 10^{-4}$, we obtained: $1.5424 \mu \mathrm{m}$ as $\lambda_{B}, 1 \mathrm{pm} / \mu \varepsilon$ and $10 \mathrm{pm} /{ }^{\circ} \mathrm{C}$ as the strain and temperature sensitivities, and $100 \%$ as the reflectivity. These results are very acceptable values if we compare them with those published in 
$[8,9]$. In addition, the proposed design is efficient, and there is no need for the apodized function of correction or for minimize sidelobs in the spectrum reflectivity. This is very important for increasing the multiplexing solutions and enhancing the used bandwidth.

To describe the behavior of the Bragg grating inscribed in the fiber core, we use the famous coupled modes theory [10]. This theory allows us to characterize the FBG and calculate its reflectivity. To apply this theory, we assume that we have an electric field that propagates in the forward and

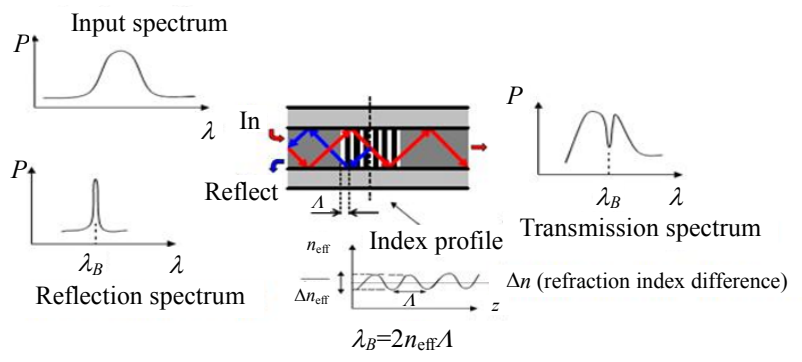

Fig. 1 Operation principle of the FBG technology and thus its response in transmission and reflection.

backward directions in the FBG. So the coupling is caused by the interaction of the electric fields, and the FBG grating can be expressed as

$$
\begin{aligned}
& \frac{d A_{1}}{d z}=\mathrm{i} \sigma(z) A_{1}(z) \exp \left(\frac{\mathrm{i} 2 \pi n_{\mathrm{eff}} z}{\lambda}\right)+\mathrm{i} K(z) A_{2}(z) \exp \left(\frac{-\mathrm{i} 2 \pi n_{\mathrm{eff}} z}{\lambda}\right) \\
& \frac{d A_{2}}{d z}=-\mathrm{i} \sigma(z) A_{2}(z) \exp \left(\frac{-\mathrm{i} 2 \pi n_{\mathrm{eff}} z}{\lambda}\right)-\mathrm{i} K(z) A_{1}(z) \exp \left(\frac{\mathrm{i} 2 \pi n_{\mathrm{eff}} z}{\lambda}\right)
\end{aligned}
$$

where $A_{1}$ is the electric field in the forward direction, $A_{2}$ is that in the backward direction, $\sigma(z)$, and $K(z)$ is the coupling coefficients either in the direct current (DC) status or in the alternating current (AC) status, respectively, along the Bragg grating length. $K(z)$ determines the power exchange between the propagation modes in the FBG. We use the matrix solution in (3) and (4) to calculate the reflected spectrum and the transmission spectrum, in which the length $L$ of the FBG is divided into $N$ uniform Bragg grating sections. Each has a length much longer than the nominal grating period. Moreover, each uniform section is represented by a square matrix $C_{i}$, and by multiplying these entire matrixes, a general matrix that describes the propagation through the full grating is obtained:

$$
\left[\begin{array}{c}
A_{1, N} \\
A_{2, N}
\end{array}\right]=C_{N} \ldots C_{i} \ldots C_{2}\left[\begin{array}{l}
A_{1,1} \\
A_{2,1}
\end{array}\right] .
$$

It should be noted that $A_{1}(z=0)=1$ is the electric field in the forward direction at the boundary limit, and $A_{2}(z=L)=0$ is that of the electric field in the backward direction at the boundary limit. The matrix $C_{i}$ for each uniform Bragg section is expressed as

$B_{i}=\left[\begin{array}{l}\cosh \left(\sqrt{k_{i}-\sigma_{i}} L_{u}\right)-\mathrm{i} \frac{\sigma_{i}}{\sqrt{k_{i}-\sigma_{i}}} \sinh \left(\sqrt{k_{i}-\sigma_{i}} L_{u}\right) \\ \mathrm{i} \frac{\pi \delta n_{\text {eff }}(z)}{\sqrt{k_{i}-\sigma_{i}} \lambda} \sinh \left(\sqrt{k_{i}-\sigma_{i}} L_{u}\right)\end{array}\right.$

$$
\begin{aligned}
& -\mathrm{i} \frac{\pi \overline{\delta n_{\text {eff }}(z)} v}{\sqrt{k_{i}-\sigma_{i}} \lambda} \sinh \left(\sqrt{k_{i}-\sigma_{i}} L_{u}\right) \\
& \cosh \left(\sqrt{k_{i}-\sigma_{i}} L_{u}\right)+\mathrm{i} \frac{\sigma_{i}}{\sqrt{k_{i}-\sigma_{i}}} \sinh \left(\sqrt{k_{i}-\sigma_{i}} L_{u}\right)
\end{aligned}
$$

where $\sigma_{i}$ and $k_{i}$ are the coupling coefficients in the direct current (DC) status and alternating current (AC) status, respectively, for each uniform section. And so the reflectivity can be expressed as

$$
R=\left(\frac{A_{2,1}}{A_{1,1}}\right)^{2} .
$$

On the other hand, to obtain the hydrogen FBG sensor, we use hydrogen sensitive film around the FBG length, as shown in Fig. 2. In addition, we can see that we have two cases: one without the etched cladding and the other with the etched cladding. Moreover, we can find various materials used as the hydrogen sensitive film like the palladium (Pd) [11, 
12], platinum (Pt) [13], and Pt-loaded $\mathrm{WO}_{3}$ (tungsten trioxide) [14]. In addition in [15], the authors used the $\mathrm{Pd} / \mathrm{Ni}$ composite film as the sensing material where the proposed sensors showed good results. In addition in [3], we can find almost all the materials used as the sensing film. On the other hand, in a previous work in [16] of our research group, we have shown that the use of the etched cladding technical increases the efficiency of the hydrogen FBG sensor, as shown in Fig. 2(b). This idea was verified also in [15]. In this paper, we want to increase the published specifications of the hydrogen FBG sensors of our research group in [4] and [16]: the sensitivity, response time, and life time, in which we used the palladium (Pd) as the hydrogen sensitive film. With these works, we had the sensitivity of $60 \mathrm{pm} / 1 \% \mathrm{H}_{2}$ and the faster response time, less than $9 \mathrm{~s}$, but with a shorter lifetime, i.e. the low repeatability of measurement. So we wanted to enhance these specifications for locating the leakage position of hydrogen more quickly and accurately to minimize the occurrence of tragedy due to the hydrogen leakage with the further repeatability. For this, we made a new design of the hydrogen FBG sensor, as shown in Fig. 3. The effectiveness of this design has been verified in [4]. However, in this paper, we use the alliance of $\mathrm{Pd}-\mathrm{Ag}$ $(500 \mathrm{~nm})$ as the hydrogen sensitive film rather than the Pd only. The aim of the use of the Ag material is to enhance the sensitivity and life time of the hydrogen sensitive film of Pd, so that of the sensor. And from the authors' knowledge, this alliance was never proposed before as the hydrogen sensitive film with the technology of the FBG. In addition, a titanium (Ti) layer $(35 \mathrm{~nm})$ was used as the adhesive layer to enhance the strength between the $\mathrm{Pd}-\mathrm{Ag}$ layer and optic cladding. And to further enhance the sensitivity of the sensor, we inscribed two other $\mathrm{Pd}-\mathrm{Ag}$ layers with a length of $4 \mathrm{~mm}$ each, in both sides of the Bragg grating. In this way, the increased sensitivity was guaranteed like presented in [4].

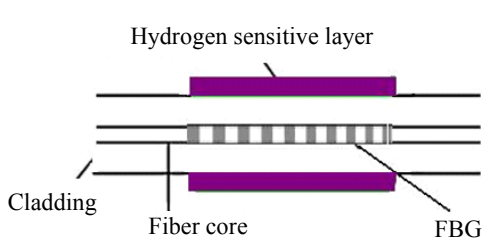

(a)

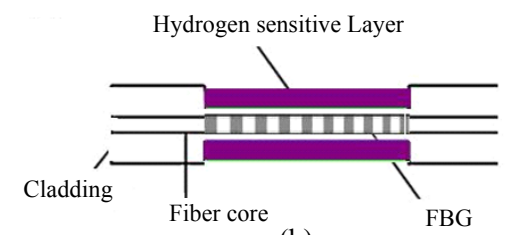

(b)

Fig. 2 Illustration of the hydrogen FBG sensor: (a) without the etched cladding and (b) with the etched cladding.

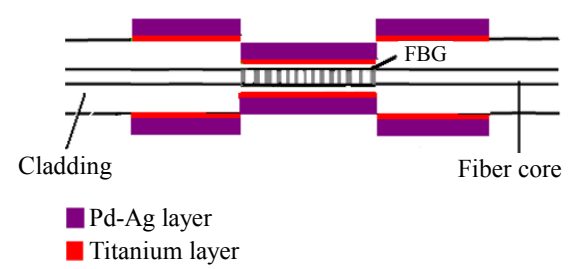

Fig. 3 Shape of the proposed hydrogen FBG sensor.

Practically, we can use a single-mode optical fiber, SMF-28. The radius of the fiber core is equal to $4.6 \mu \mathrm{m}$, that of the optic cladding is $62.5 \mu \mathrm{m}$, and that of the mechanical protective cladding is $125 \mu \mathrm{m}$. Figure 4 shows the picture of 72 optical fibers with the type mentioned above, and Fig. 5 shows the picture of two optical fibers. And to write our special FBG, firstly we inscribed a uniform FBG with the grating period equal to $0.5284 \mu \mathrm{m}$, and then we used carefully the stretch movement in the two sides of the FBG, in order to obtain the shape mentioned above and according to (2). In addition, our sensor was defined with the etched cladding with a radius of $62 \mu \mathrm{m}$, so the remainder of the cladding layer along the FBG was $0.5 \mu \mathrm{m}$. Figure 5 gives a picture of two optical fibers with the characteristics mentioned above. The first is the normal fiber, and the second is the optical fiber with the etched cladding with a radius of the $62 \mu \mathrm{m}$ and a length of $4 \mathrm{~mm}$ in the practical way. Thereafter above the remainder cladding, about $0.5 \mu \mathrm{m}$, the Pd-Ag layer $(500 \mathrm{~nm})$ would be carried out, as shown in Fig. 3. Thus, the wavelength shift of our FBG hydrogen sensor had a relationship with the 
value of the etched cladding and the thickness of the Pd-Ag film. However, the thickness of Pd-Ag was selected to have a maximum strain which would be applied to the length of our hydrogen FBG sensor with the minimum effect of the peeled off phenomenon with the cladding, while the value of the etched cladding with a radius of $62 \mu \mathrm{m}$ was selected to have also the maximum strain and as well to have the minimum level of protection required for the fiber core. In addition, it is obvious that the use of the etched cladding technically increases the performances of our sensor.

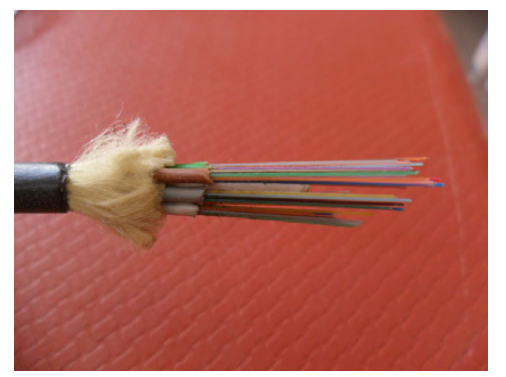

Fig. 4 Picture of 72 optical fibers with the type SMF-28.

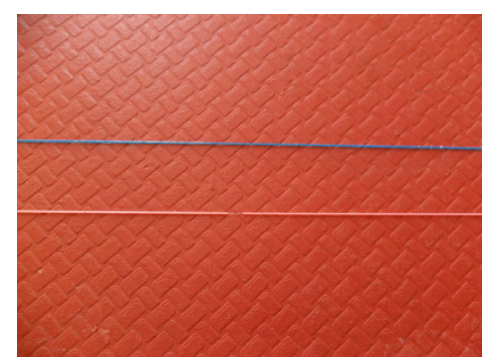

Fig. 5 Picture of two used optical fibers with the type SMF-28: the first is the normal optical fiber, and the second is the optical fiber with the etched cladding $(62 \mu \mathrm{m})$ with a length of $4 \mathrm{~mm}$ in the middle.

On the other hand, the preparation of the Pd-Ag layer can be realized with the sol gel method, evaporation method, etc. And to avoid the Pd-Ag coating layer from being peeled off when we had a high hydrogen concentration, we used the adhesive layer below the Pd-Ag layer. In our case, this adhesive layer was the titanium (Ti) layer with the low thickness $(35 \mathrm{~nm})$. Therefore, our hydrogen FBG sensor consisted of the fiber core, fiber cladding $(0.5 \mu \mathrm{m})$, Ti layer $(35 \mathrm{~nm})$, and Pd-Ag layer $(500 \mathrm{~nm})$. Moreover, in practical cases, we advise for both the Pd-Ag layer and the Ti layer to coat them by using magnetron sputtering where $\mathrm{Pd}-\mathrm{Ag}$ and $\mathrm{Ti}$ grains would be evenly distributed. So we can have high quality uniform metal layers in compared to the other classic coating technologies, which protect the FBG and improve also the sensor's sensitivity. This idea is confirmed also in [17].

\section{Results and discussion}

\subsection{Results}

The behavior of our FBG sensor remains the same using the Pd-Ag coating technique if we have no disruptive effect exerted on the FBG length. However, the thickness of the Pd-Ag layer is the optimal thickness used to achieve the performance characteristics, i.e. the desired stress exerted on the FBG length. Moreover, Fig. 6 gives the experimental model used to examine our hydrogen sensor. This experimental model has been implanted with the necessary components in OptiSystem software from Optiwave Society, Canada and in LabVIEW software from National Instruments Society, USA. The gas test cell had two gates for the gas flow in and out. The hydrogen gas flow rate was measured and controlled separately by the flow meter, where the varying hydrogen concentrations were provided by changing the flowing rate of $\mathrm{H}_{2}$. And to investigate the influence of variant hydrogen concentrations on the shift in the FBG wavelengths, the FBG sensor array was inserted into the gas cell. Two rubber corks, easy penetration from the middle, were fixed in a gas cell sides to facilitate the implantation of the FBG sensors array inside the gas cell and to minimize any hydrogen leakage in point in and in point out of the FBG sensors array in the gas cell. The wavelength of the laser light source was equal to $1.5 \mu \mathrm{m}(199.8 \mathrm{THz})$. An optical coupler with a loss of $3 \mathrm{~dB}$ was used for combining or splitting optical signals. An investigation system was used to calculate the shift in the wavelength. It consisted of an amplification component used to amplify the reflected signal, a band-pass filter used 
to filter the signal from the amplify component, and a PC with the LabVIEW program used to analyze, process, and save the data in real time. This investigation system has the same function with the optical spectrum analyzer (OSA). In addition, the FBG sensor array contained our FBG hydrogen sensor and a temperature compensation FBG sensor. The temperature compensation FBG sensor was used to eliminate the effect of temperature when calculating the shift of the FBG hydrogen sensor. So we selected the temperature sensitivity of the temperature compensation FBG sensor to be equal to that of our FBG hydrogen sensor. For us, the central wavelength of the hydrogen FBG sensor was set at $1.5424 \mu \mathrm{m}$, as we have seen before, whereas we fixed the central wavelength of the temperature compensation sensor at $1.5563 \mu \mathrm{m}$. On the other hand, the practical experiment of such system can be performed at the room temperature of $25^{\circ} \mathrm{C}$ and at the atmospheric pressure but taking precautionary measures for the rescue reason. For us, we have also performed the components in LabVIEW and OptiSystem softwares at $25^{\circ} \mathrm{C}$.

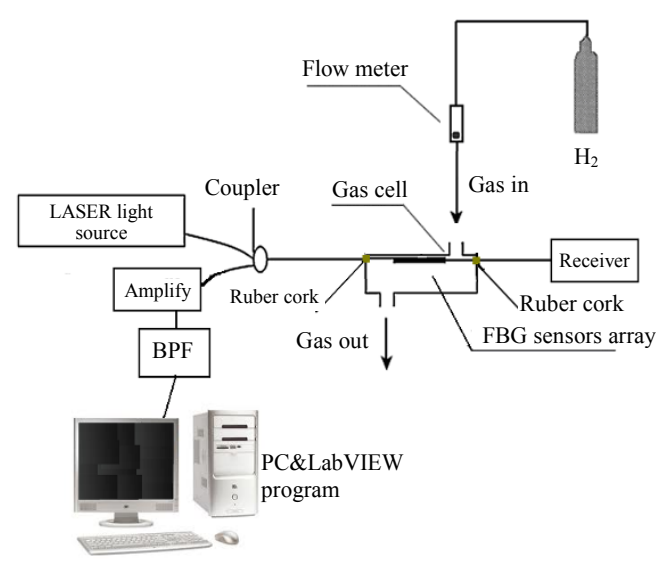

Fig. 6 Hydrogen sensing experimental model.

\subsection{Discussion}

As operation, the Pd-Ag layer absorbed hydrogen, thereby a strain change was induced in the FBG length. Consequently, the shifts in the wavelength were realized. These shifts were observed with different hydrogen concentrations.

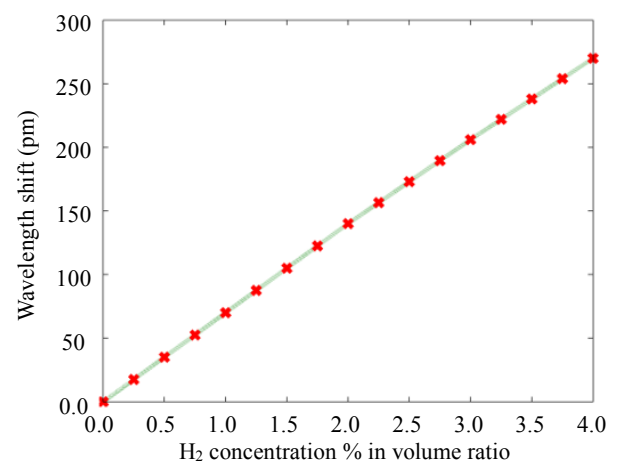

Fig. 7 Wavelength shifts of the proposed hydrogen FBG sensor in the $0.1 \% \mathrm{H}_{2}-4 \% \mathrm{H}_{2}$ range in the volume ratio.

Figure 7 indicates these shift responses after eliminating the temperature effect using the data in the LabVIEW program in the $0.1 \% \mathrm{H}_{2}-4 \% \mathrm{H}_{2}$ range. Our FBGs had the temperature sensitivity in the order of $10 \mathrm{pm} /{ }^{\circ} \mathrm{C}$ at the room temperature. From the curve in Fig. 7, the wavelengths increase almost linearly with a function of the hydrogen concentration. In addition, the results showed the highest sensitivity. In the $0.1 \% \mathrm{H}_{2}-2 \% \mathrm{H}_{2}$ range, the sensitivity reached $70 \mathrm{pm} / 1 \% \mathrm{H}_{2}$, and in $2 \% \mathrm{H}_{2}-$ $4 \% \mathrm{H}_{2}$ range, this sensitivity was about $65 \mathrm{pm} / 1 \% \mathrm{H}_{2}$ $-70 \mathrm{pm} / 1 \% \mathrm{H}_{2}$. These obtained sensitivities are the better sensitivities obtained up to now according to our knowledge in compared to those published. In [14] and [15], the authors published the resolution equal to $60 \mathrm{pm} / 1 \% \mathrm{H}_{2}$ in the $0.1 \% \mathrm{H}_{2}-2 \% \mathrm{H}_{2}$ range. Also, we have confirmed, by ourselves, this resolution in the previous work in [4]. On the other hand, to well characterize our FBG sensor, we have calculated its response and recovery time in the case of the presence of hydrogen. The response time gave the completed time for reaching the maximum wavelength shift in the presence of quantity of hydrogen. Practically, it can be calculated from the moment of hydrogen flowing into the gas room to the moment of the FBG reaching the maximum wavelength shift relative to a reference wavelength of the FBG sensor. For our sensor, the response time is faster than that in the published literature up to now, so is the resolution, as shown in Fig. 8. The response time of our sensor was about $4 \mathrm{~s}-5 \mathrm{~s}$ for each $1 \% \mathrm{H}_{2}$. Whereas in [14], the authors published 


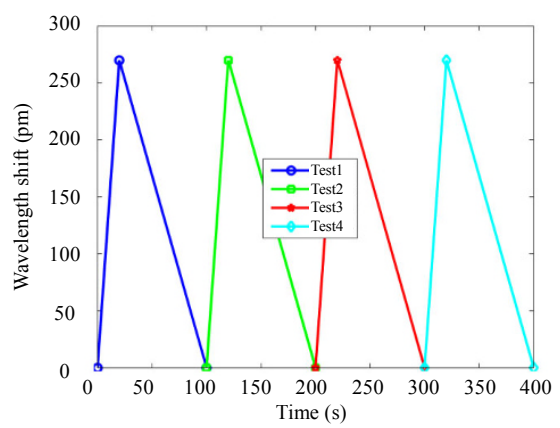

Fig. 8 Four tests of the response time and recovery time of the hydrogen FBG sensor in the range of $0.1 \% \mathrm{H}_{2}-4 \% \mathrm{H}_{2}$ and the normal air.

a response time equal to $10 \mathrm{~s}$. Also, we have found a response time equal to $6 \mathrm{~s}$ in [4]. In addition, from the curve in Fig. 8, we had about 20s as a response time in the presence of $4 \% \mathrm{H}_{2}$, which means four-times response time for each $1 \% \mathrm{H}_{2}$. This result indicated that the response time was almost fixed in the range of $0.1 \% \mathrm{H}_{2}-4 \% \mathrm{H}_{2}$ for every step of $1 \% \mathrm{H}_{2}$. And this is absolutely a performance of the stability. Moreover, if we minimize the response time, we can increase absolutely the rescue time for the equipments and people if any hydrogen leakage occurs. In addition, the recovery time is faster, also as indicated in Fig. 8. It was equal to $80 \mathrm{~s}$ in the presence of $4 \% \mathrm{H}_{2}$ in four tests, whereas in [14] the authors published a recovery time equal to $120 \mathrm{~s}$ in the presence of $4 \% \mathrm{H}_{2}$. Practically, this recovery time was calculated from the moment of the normal air flowing into the gas room where the maximum shift wavelength was reached to the moment where the FBG wavelength returned to normal. In addition, from Fig. 8, it is clear that the repeatability is very precise in the case of the response time and recovery time. This is very important, by which the life time of the FBG sensor is enhanced. Also, this is a stability index for the sensor. This is due essentially to the presence of the Ag material in the hydrogen sensitive film of Pd. In addition, it can be seen that the fast response time, $4 \mathrm{~s}-5 \mathrm{~s}$ for each $1 \% \mathrm{H}_{2}$, is attributed to this material, which minimizes the hydrogen absorption time in the crystal lattice of Pd. Also, it helps to decrease rapidly the complex refractive index of the $\mathrm{Pd}$ with an increase in the absorption of hydrogen.

Considering the resolution of $1 \mathrm{pm}$ for the investigation system, $0.014 \% \mathrm{H}_{2}$ can be detected in air by our special FBG sensor in the range of $0.1 \% \mathrm{H}_{2}-4 \% \mathrm{H}_{2}$. So it was reported, from the obtained results, that our FBG hydrogen sensor coated with $\mathrm{Pd}-\mathrm{Ag}$ presented the highest sensitivity with the linear response, faster response time, and the further repeatability when the hydrogen concentration was less than the explosive limit $\left(4 \% \mathrm{H}_{2}\right.$ in the volume ratio). On the other hand, practically the FBG hydrogen sensor can be a combustion source when the hydrogen concentration is more than $4 \% \mathrm{H}_{2}$ in air and the temperature around the FBG increases to more than $100{ }^{\circ} \mathrm{C}$. Therefore, to obtain the FBG hydrogen sensor with the intrinsic safety, the temperature which will influence the concentration of $\mathrm{H}_{2}$ around the FBG should be controlled. Otherwise, in several applications, the hydrogen concentration may be more than $4 \%$ in the volume ratio. Figure 9 illustrates the response of our special FBG sensor at the higher hydrogen concentration using the data in the LabVIEW program. From the curve in Fig. 9, when the hydrogen concentration increases, the FBG wavelength shifts to the longer wavelength, but in a slow manner with a nonlinear response. And owing to the structure of the FBG, when the hydrogen increases to $50 \%$, which is the highest value,

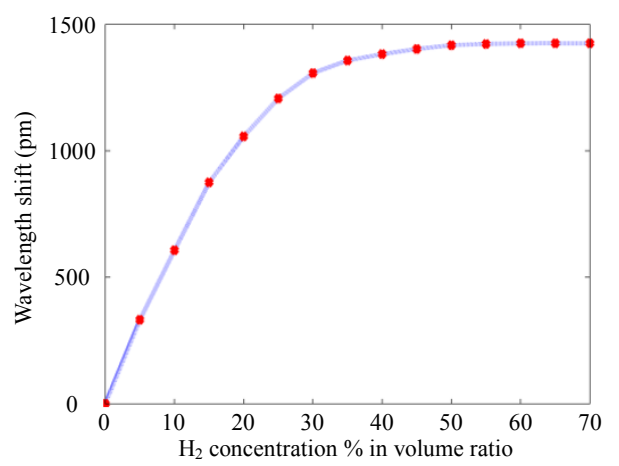

Fig. 9 Wavelength shifts of the proposed hydrogen FBG sensor at the high hydrogen concentrations. 
published up to now [4, 14], the Pd-Ag will be almost totally consumed, and the central wavelength will be fixed. Despite this result, the proposed FBG sensor is efficiency even under almost the pure hydrogen environment. Subsequently, we hope that it can be used to monitor easily any hydrogen leakage.

\section{Application in propulsion system fuel tank of aerospace vehicle}

The type of the hydrogen FBG sensor is very promising for the disaster aerospace prevention. In addition, using the wavelength division multiplexing (WDM) technique, the total body of an aerospace vehicle recovery against the hydrogen leakage is guaranteed, particularly in the components which are most sensitive to hydrogen such as propulsion system fuel tanks, feed lines, and engine elements. And from the obtained results in the first parts of this paper, we have applied 3 hydrogen FBG sensors proposed by us in the propulsion system fuel tank model using a selector technique rather than the WDM technique, i.e. FBG1, FBG2, and FBG3, which have the same wavelength: $\lambda_{B}=1.5424 \mu \mathrm{m}$. In addition, 3 temperature compensation FBG sensors were used to compensate the temperature effect in the calculation of the wavelength shifts, i.e. FBG4, FBG5, and FBG6. Each temperature compensation FBG sensor was used with each FBG hydrogen sensor. And as there is no possibility of having a real propulsion system fuel tank of an aircraft or an aerospace vehicle, as shown in Fig. 10, we used a model of a bottle in aluminum (Al) filled with $3 \%$ hydrogen and $97 \%$ nitrogen (N2). The use of the aluminum material as the wall material of the bottle has a high safety during the storage of hydrogen at the high pressure. In addition, we can use the austenitic steel or a glass in place of the aluminum. The installation model of this proposed propulsion system fuel tank, with 3 hydrogen FBG sensors and 3 temperature compensation FBG sensors, is special. The first couple, FBG1 and
FBG4, was installed in the aft dome. The second couple, FBG2 and FBG5, was installed above the cylinder portion, and the third couple, FBG3 and FBG6, was installed in the forward dome. Figure 11 gives an illustration of a part of the installed system, in which we used a selector to select at a specific moment one of the three sensors: a dispersion compensator which is related to the length of the fiber, a mix, a photo detector, and two amplifiers one: optic and the other: electric.

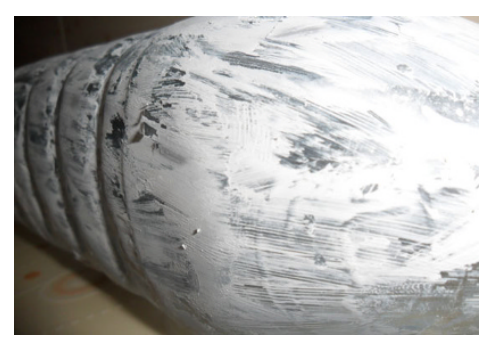

Fig. 10 Illustration of the real propulsion system fuel tank model.

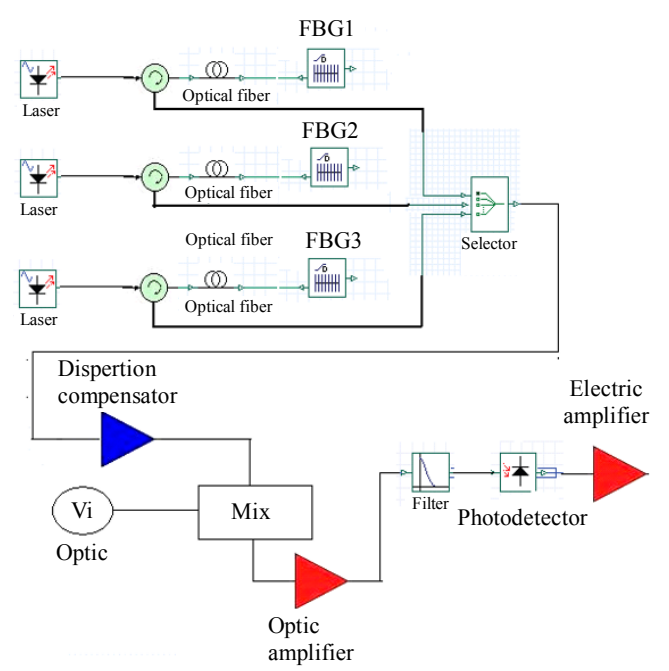

Fig. 11 Installed system of three hydrogen sensors in the propulsion system fuel tank model.

We have investigated the response of the sensor systems with real-time monitoring. In the absence of any leakage hydrogen, the central wavelengths of three hydrogen FBG sensors remained the same. And there was no possibility of hydrogen leak because our propulsion system fuel tank remained motionless, and it was a model type. We are looking for a way to have the artificial hydrogen leakage. And by the realization of the leakage in the cylinder portion, we had the curves shown in Fig. 12. We 
used the same reasoning like that of heat diffusion with some modifications. From these curves, FBG2 detects $1 \%$ of the hydrogen leakage in $4 \mathrm{~s}$, and FBG1 and FBG3 detect this leakage concentration in $6 \mathrm{~s}-$ $7 \mathrm{~s}$. These results are very normal because the vanishing point is located next to the sensor FBG2, while the other sensors are a bit far from this vanishing point. So the presence of the sensor near the vanishing point is very important to have a faster response and a precise localization. Consequently, our proposed system is effective to detect hydrogen leakage in the propulsion system fuel tank of the aerospace vehicle.

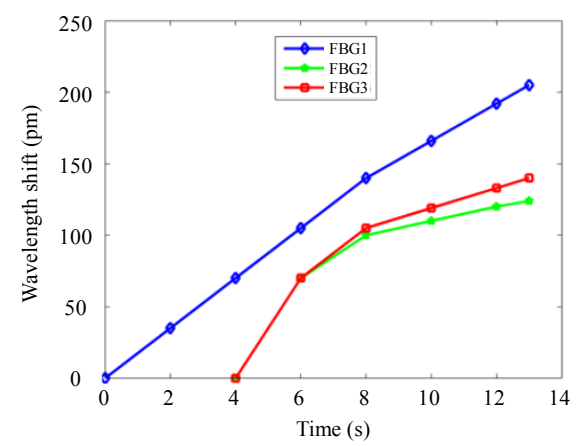

Fig. 12 Responses of FBG1, FBG2 and FBG3 to hydrogen leakage in the propulsion system fuel tank model of the aerospace vehicle.

On the other hand, each sensor must be calibrated. For our sensor, we have calibrated the shift values for $0.1 \%-5 \% \mathrm{H}_{2}$ in the range of $0.5 \mathrm{~V}-$ $5 \mathrm{~V}$. The expression that describes this calibration is

$$
V=1.631 * C_{\mathrm{H}_{2}}{ }^{-066} \text {. }
$$

This expression is obtained with a simple circuit that is implanted in the ISIS software from Labcenter Electronics Society, where $V$ is the obtained voltage, and $C_{\mathrm{H}_{2}}$ is the hydrogen concentration.

Figure 13 gives an illustration curve of this equation. And it is clear from the curve that when the obtained voltage is below $1 \mathrm{~V}$, this means that the hydrogen concentration is more than $3 \%$. In addition, to cover a total body of the most sensible components to hydrogen leakage of an aerospace vehicle, we use the same reasoning with the WDM. Therefore, our special proposed hydrogen FBG sensor can be used for disaster environment prevention in which locating the fault position of hydrogen is more quickly and accurately than the conventional and is essential to save people and materials, especially in the sensitive environment and in the environment where the hydrogen presents. Otherwise, we have demonstrated theoretically that our special FBG hydrogen sensor, using the etched cladding with the $\mathrm{Pd}-\mathrm{Ag}$ coating as the hydrogen sensitive film, is very efficiency. This is due essentially to the presence of the Ag material.

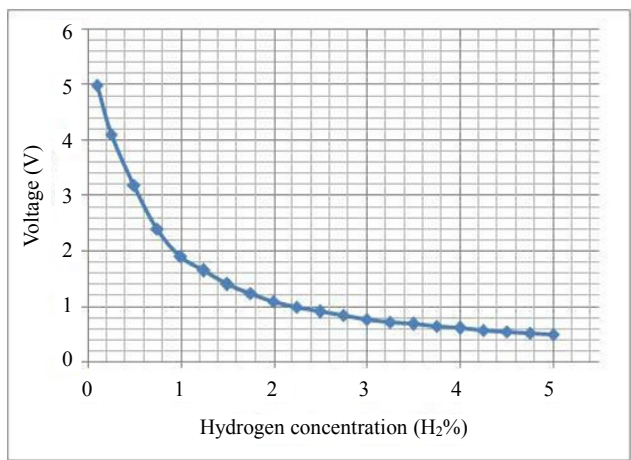

Fig. 13 Calibration curve of the hydrogen sensor.

\section{Conclusions}

A new FBG hydrogen sensor with a length of $4 \mathrm{~mm}$ based on the alliance Pd-Ag material $(500 \mathrm{~nm})$ as the hydrogen sensitive film and the Ti layer (35 $\mathrm{nm})$ as the adhesive layer was developed. Its sensing characteristics have been investigated. From the simulation results, the better results were obtained especially in the low concentration range of hydrogen, where we had the resolution reaching $70 \mathrm{pm} / 1 \% \mathrm{H}_{2}$ and the faster response time in the order of $4 \mathrm{~s}-5 \mathrm{~s}$. In addition, the repeatability was guaranteed. These results are due especially to the presence of the $\mathrm{Ag}$ material in the hydrogen sensitive film. And with the FBG demodulator resolution of $1 \mathrm{pm}$, a high precision to the slight hydrogen concentration variation can be detected: $0.014 \% \mathrm{H}_{2}$. Also it is demonstrated that the sensor is safe even at the higher hydrogen concentration. Therefore, we hope that with the Pd-Ag alliance, the sensor has more resistant to damage from exposure 
to the high hydrogen concentration than the Pd only with very faster response time than those of all the solutions presented and published up to now in leakage hydrogen detection with the FBG technology. Otherwise, this sensor is very promising for the disaster aerospace prevention in which locating the leakage position of hydrogen more quickly and accurately is very important to save people and equipments. In addition, with the selector technique, the body of the propulsion system fuel tank of the aerospace vehicle recovers against hydrogen leakage with 3 hydrogen FBG sensors proposed by us which was demonstrated theoretically. The results were investigated in real time, and we hope that we have developed a disaster aerospace prevention system against hydrogen leakage using the FBG technology, especially in the unmanned flight with a long duration. In addition, the results mentioned above can be applied in other areas such as in the pipe walls or nuclear fuel cladding. So there are more scopes for development. Moreover, all experiments made based on the results in this paper should be with precaution for the rescue reasons, since hydrogen is very dangerous in the normal closed places, but we hope that the results to be obtained will confirm those published in this paper.

\section{Acknowledgment}

This work was supported by the laboratory of Advanced Materials and Quantum Phenomena, Faculty of Sciences of Tunis, Tunis-EL Manar University in 2013-2014 funded by the Ministry of Higher Education and Scientific Research, Tunisia.

Open Access This article is distributed under the terms of the Creative Commons Attribution License which permits any use, distribution, and reproduction in any medium, provided the original author(s) and source are credited.

\section{References}

[1] X. Bevenot, A. Trouillet, C. Veillas, H. Gagnaire, and
M. Clément, "Hydrogen leak detection using an optical fiber sensor for aerospace application," Sensors and Actuators B: Chemical, 2000, 67(1-2): 57-67.

[2] S. K. Mital, J. Z. Gyekenyesi, S. M. Arnold, R. M. Sulliven, J. M. Mandersceid, and P. L. N. Murthy, "Review of current state of the art and key design issues with potential for liquid hydrogen crygenic storage tank structures for aircraft applications," NASA Technical Reports Server, 2006, Document ID: 20060056194.

[3] T. Hûbert, L. Boon-Brett, G. Black, and U. Banach, "Hydrogen sensors-A review," Sensors and Actuators B: Chemical, 2011, 157(2): 329-352.

[4] S. Saad and L. Hassine, "Hydrogen detection with FBG sensor technology for disaster prevention," Photonic Sensors, 2013, 3(3): 214-223.

[5] G. W. Hunter, D. B. Makel, E. D. Jansa, G. Patterson, P. J. Cova, C. C. Liu, et al., "A hydrogen leak detection system for aerospace and commercial application," SAO/NASA ADS Physics Abstract Service, 1995, AIAA-95-2645.

[6] K. O. Hill and G. Meltz, "Fiber Bragg grating technology fundamentals and overview," Journal of Lightwave Technology, 1997, 15(8): 1263-1276.

[7] A. D. Kersey, M. A. Davis, H. J. Patrick, M. LeBlanc, K. P. Koo, C. G. Askins, et al., "Fiber grating sensors," Journal of Lightwave Technology, 1997, 15(8): 1442-1463.

[8] D. H. Kang, S. O. Park, C. S. Hong, and C. G. kim, "The signal characteristics of reflected spectra of fiber Bragg grating sensors with strain gradients and grating length," NDT\&E International, 2005, 38(8): 712-718.

[9] Q. Wu, G. Farrell, and Y. Semenova, "Simple design technique for a triangular FBG filter based on a linearly chirped grating," Optics Communications, 2010, 283(6): 985-992.

[10] C. Lu, J. Cui, and Y. Cui, "Reflection spectra of fiber Bragg grating with random fluctuations," Optical Fiber Technology, 2008, 14(2): 97-101.

[11] B. Sutapun, M. Tabib-Azar, and A. Kazemi, "Pd-coated elastooptic fiber optic Bragg grating sensors for multiplexed hydrogen sensing," Sensors and Actuators B: Chemical, 1999, 60(1): 27-34.

[12] C. Tien, H. Chen, W. Liu, S. Jyu, S. Lin, and Y. Lin, "Hydrogen sensor based on side-polished fiber Bragg gratings coated with this palladium film," Thin Solid Films, 2008, 516(16): 5360-5363.

[13] L. Goddard, K. Y. Wong, A. Garg, E. Behymer, G. Cole, and T. Bond, "Measurement of the complex refractive index of $\mathrm{Pd}$ and $\mathrm{Pt}$ films in air and upon adsorption of $\mathrm{H}_{2}$ gas," in 21st Annual Meeting of the IEEE Lasers and Electro-Optics Society 2008, Acapulco, Mexico, Nov. 9-13, pp. 569-570, 2008.

[14] Mi. Yang, Z. Yang, J. Dai, and D. Zhang, "Fiber 
optic hydrogen sensors with sol-gel $\mathrm{WO}_{3}$ coatings," Sensors and Actuators B: Chemical, 2012, 166-167: 632-636.

[15] J. Dai, M. Yang, X. Yu, K. Cao, and J. Liao, "Greatly etched fiber Bragg grating hydrogen sensor with $\mathrm{Pd} / \mathrm{Ni}$ composite film as sensing material," Sensors and Actuators B: Chemical, 2012, 174: 253-257.

[16] Said Saad and Lotfi Hassine, "Hydrogen detection with inverted chirped FBG sensor for disaster environment prevention," in IEEE Conference, 10th International Multi-Conference, Systems, Signals \& Devices (SSD'13), Hammamet, Tunisia, Mar. 18-21, pp. 1-6, 2013.

[17] G. Ma, C. Li, Y. Luo, R. Mu, and L. Wang, "High sensitive and reliable fiber Bragg grating hydrogen sensor for fault detection of power transformer," Sensors and Actuators B: Chemical, 2012, 169, 195-198. 